\title{
Internet das Coisas como Plataforma Multidisciplinar de Aprendizagem: Projetos Integradores Hardware/Software
}

\author{
Vinícius F. S. Mota ${ }^{1}$, Roberta L. Gomes ${ }^{1}$, Sérgio A. A. de Freitas ${ }^{2 *}$ \\ ${ }^{1}$ Universidade Federal do Espírito Santo (UFES) - Vitória/ES - Brasil \\ ${ }^{2}$ Universidade de Brasília (UnB) - Brasília/DF - Brasil \\ vinicius.mota@inf.ufes.br, roberta.gomes@ufes.br, sergiofreitas@unb.br
}

\begin{abstract}
This paper presents an experience report of the development of a teaching project, entitled "Internet of Things as a multidisciplinary learning platform ", and a case study of the artifacts resulting in an integrated project course. This project provided technical-didactic material discussing the Internet of Things, integrating hardware/software/ communication. Thus, the students were exposed to advanced and practical concepts in the second semester of the Computer Engineering course at the Federal University of Espírito Santo. Through an intrinsic motivation test, we observed a high level of interest and self-confidence among students to perform the course's tasks.
\end{abstract}

\begin{abstract}
Resumo. Este trabalho apresenta um relato de experiência do desenvolvimento de um projeto de ensino, intitulado "Internet das Coisas como plataforma multidisciplinar de aprendizagem”, e um estudo de caso da aplicação dos artefatos resultantes em uma disciplina de projeto integrado. Tal projeto disponibilizou material técnico-didático abordando a temática Internet das Coisas, integrando hardware/software/comunicação. Dessa forma, os discentes foram expostos a conceitos avançados e práticos já no segundo período do curso de Engenharia de Computação da Universidade Federal do Espírito Santo. Por meio de um teste de motivação intrínseca, observou-se um alto índice de interesse e autoconfiança dos alunos na elaboração das tarefas da disciplina.
\end{abstract}

\section{Introdução}

Em 2018, os cursos de Ciência da Computação e Engenharia da Computação ficaram em sexto e trigésimo lugar, respectivamente, em porcentagem de alunos que deixaram o curso sem se formar nas universidades públicas brasileiras [INEP 2019]. Diversos fatores estão associados à evasão universitária, tais como, insatisfação, questões financeiras, deficiências de aprendizagem, desmotivação com o curso, entre outros [Davok and Bernard 2016]. Por meio de entrevistas, um estudo realizado pela UFPE mostra que os principais fatores individuais dos estudantes que evadiram os cursos da área de ciências exatas e tecnologia foram incompatibilidade da vida acadêmica com o mercado de trabalho e desmotivação/falta de identidade com o curso [UFPE 2016].

Diante dessa problemática, foi proposto um projeto de ensino, intitulado "Internet das Coisas como plataforma multidisciplinar de aprendizagem". A escolha da temática Internet das Coisas deve-se a dois motivos: i) $\mathrm{O}$ aumento de objetos conectados à Internet; ii) integração de hardware e software. Internet das Coisas, IoT na sigla em inglês,

\footnotetext{
* Os autores agradecem à CAPES, CNPq, FAPES e UFES pelo apoio financeiro parcial a este trabalho.
} 
é o domínio de aplicação que integra diferentes campos tecnológicos, como tecnologias de sensoriamento, novos protocolos de redes e novos serviços [Minerva et al. 2015]. Portanto, projetos de IoT são intrinsecamente multidisciplinares.

Este artigo apresenta um relato de experiência da realização deste projeto para integrar o desenvolvimento entre software e hardware, habilidade obrigatória para profissionais de Computação. Os resultados desse projeto foram roteiros de aulas práticas a serem utilizados em disciplinas integradoras, com base na abordagem de aprendizado baseado em problema (PBL) [Duch et al. 2001]. Para validar os resultados, o material desenvolvido foi utilizado na disciplina "Projeto Integrado de Computação I", no segundo período do curso de Engenharia de Computação da UFES em Vitória. Ao término da disciplina, os estudantes realizaram uma avaliação motivacional, usando o Índice de Motivação Intrínseca (IMI), que permitiu a análise de como projetos de software/hardware realizados no início do curso podem aumentar a motivação nos cursos de computação.

O restante do artigo está organizado como segue. A Seção 2 descreve o projeto de ensino. O estudo de caso utilizando os artefatos do projeto é apresentado na Seção 3. A avaliação de motivação intrínseca dos estudantes é discutida na Seção 4. Por fim, a Seção 5 conclui e discute os trabalhos futuros.

\section{Desenvolvimento do Projeto de ensino}

\subsection{Motivação}

De modo genérico, projetos IoT são compostos de três partes [Miorandi et al. 2012]: objeto "inteligente", comunicação e análise/visualização de dados. Os objetos são compostos de sistemas embarcados (System-On-Chips - SoC) e um conjunto de sensores.

O projeto de ensino permite que professores e estudantes de diversas disciplinas possam propor atividades abordando a temática de IoT, abstraindo detalhes alheios às disciplinas. Além disso, os roteiros promovem uma percepção da ligação entre as diferentes disciplinas dos cursos, podendo ser explorados em disciplinas integradoras.

De fato, as disciplinas Eletrônica, Sistemas Digitais e Sistemas Embarcados podem utilizar SoCs para exemplificar assuntos abordados nas disciplinas. Já em Redes e Sistemas Distribuídos podem ser exploradas tecnologias e protocolos para a comunicação dos dados. Nas disciplinas de Banco de Dados e Análise de dados podem ser discutidos armazenamento, visualização e análise de dados gerados pelos sensores.

\subsection{Metodologia}

O projeto foi submetido e selecionado no edital de projetos de ensino da Pró-Reitoria de Graduação da UFES em 2018. Foram selecionados dois bolsistas e um voluntario em 2019, sendo estes os responsáveis por escrever, testar e publicar os roteiros.

O desenvolvimento do projeto foi composto das seguintes etapas: i) Especificação dos roteiros de aula: Definição de aplicações Internet das Coisas, sensores utilizados, topologia de redes, linguagens de programação, softwares e o ambiente destino dos dados a serem processados para exibição e/ou atuação em um dispositivo; ii) Desenvolvimento: Implementação dos protótipos especificados. Todo o desenvolvimento seguiu a metodologia de código aberto; iii) Testes e validação: Depuração dos protótipos implementados. Em caso de detecção de problemas, o protótipo volta à etapa de desenvolvimento. Cada bolsista foi responsável por testar a solução realizada pelo outro; documentação e publicação dos protótipos; e iv) Documentação e publicação dos 
protótipos: documentação detalhando o processo de montagem, esquemático e códigos necessários para o funcionamento de cada protótipo.

\subsection{Resultados e Artefatos produzidos.}

Os resultados podem ser avaliados de forma quantitativa e qualitativa. Dos resultados quantitativos: 2 bolsistas e 1 voluntário implementaram e escreveram 18 projetos, com níveis de dificuldades evolutivos para execução de cada projeto. Qualitativamente, os resultados do primeiro ano do projeto estão disponíveis em

\section{https://gitlab.com/vmota/ensino-iot-ufes}

\section{Estudo de caso: Projeto Integrador em Computação}

No segundo semestre de 2019, de forma piloto, foi realizada uma disciplina Projeto Integrador de Computação I - PIC-I para o 2o período do curso de Engenharia de Computação da UFES, em que foram utilizados os resultados e artefatos produzidos no contexto do Projeto de Ensino aqui apresentado. Dessa forma, o professor pôde contar com ferramentas e tecnologias digitais para facilitar o trabalho com diferentes conteúdos de forma contextualizada [Felipe and de Melo Pinheiro 2018].

\subsection{Metodologia}

A disciplina foi divididas em três partes: i) Nivelamento - Eletrônica básica/Programação; ii) Desafios com dificuldade incremental; iii) Desenvolvimento do projeto.

No nivelamento, os estudantes foram apresentados aos conceitos básicos de eletrônica e prototipagem, tais como cálculos de valores de resistência, uso de protoboards, tipos de entrada e sáida, e às placas de desenvolvimento (SoC). Para a disciplina foram utilizadas as placas Arduino UNO e ESP 8266.

Após, os alunos tiveram que resolver problemas com dificuldades incrementais. Os problemas começaram com níveis fáceis, como desenvolvimento de um semáforo de uma e de múltiplas vias. Os problemas de nível médio requisitavam o uso de sensores e implementação de algoritmos. Por exemplo, utilizar um acelerômetro para detectar direção de movimentação (cima, baixo, direita, esquerda). A complexidade dos algoritmos foi aumentada. Por exemplo, os alunos tiveram que desenvolver um protótipo capaz de escrever uma mensagem baseado apenas na movimentação ${ }^{1}$.

Por fim, os alunos foram divididos em grupos de até três estudantes, compondo os grupos por habilidades complementares. Cada grupo propôs livremente um projeto a ser apresentado ao final da disciplina. Em cada proposta deveria constar o problema abordado, componentes necessários, etapas e cronograma de desenvolvimento. Para a apresentação final do projeto foi organizada uma "Mostra de Projetos Integradores em Computação".

\subsection{Resultados}

No total, foram elaborados sete projetos. Os grupos foram incentivados a gravar um vídeo com o resultado. Um vídeo resumo com os resultados está disponível em https://youtu.be/C2mCxcmfEXU. ${ }^{2}$

\footnotetext{
${ }^{1} \mathrm{O}$ resultado do protótipo de escrita de mensagens baseando-se nos movimentos das mãos pode ser visto em https://youtu.be/S8UybJqMzk4

${ }^{2}$ Vídeos completos em https://www.youtube.com/playlist?list=PLcQfLriE4Fbw8yW30HXIhpv1p0Ik9tQK-
} 
Por um lado, a disciplina de Projeto Integrado envolveu conhecimentos apresentados anteriormente aos alunos, como programação básica e eletrônica. Por outro lado, os projetos elaborados e desenvolvidos pelos alunos requereram técnicas e conhecimentos que ainda não haviam sido abordados em disciplinas. Com isso, os roteiros do projeto de ensino foram fundamentais para facilitar a abstração e o uso desses conhecimentos.

\section{4. Índice de Motivação}

Para avaliar a premissa de que a abordagem PBL motivou os/as estudantes foi utilizado o Intrinsic Motivation Inventory (IMI). O IMI é uma medida multidimensional para avaliar a experiência dos participantes em uma determinada atividade [Monteiro et al. 2015].

Baseado em [Monteiro et al. 2015], foram definidas 21 perguntas para mensurar quatro dimensões : Interesse (I) pela atividade; a confiança do estudante em sua competência (C); a confiança que a atividade era uma Escolha $(\mathbf{E})$; e a Pressão (P) sentida.

No IMI as perguntas de cada grupo são apresentadas em uma ordem aleatória com um valor de 1 a 7 , sendo 1 discordo totalmente e 7 concordo totalmente. A Tabela 1 mostra as perguntas na ordem apresentadas aos participantes do experimento e a dimensão (D) medida pela pergunta. As perguntas marcadas como reversas (R) têm seus valores subtraídos de 8. No IMI, cada dimensão é a média das respostas. Dos vinte estudantes matriculados na disciplina, 10 responderam de forma anônima ao questionário. Desses, quatro informaram ser do sexo feminino e seis informaram ser do sexo masculino.

\begin{tabular}{|l|c|l|}
\hline$\#$ & D & Pergunta \\
\hline 1 & I & Enquanto trabalhava na tarefa, estava pensando em quanto eu gostava. \\
\hline 2 & $\mathbf{P}$ & Eu não estava nem um pouco nervoso por fazer a tarefa. (R) \\
\hline 3 & $\mathbf{E}$ & Eu senti que era minha escolha fazer a tarefa. \\
\hline 4 & $\mathbf{C}$ & Eu era bastante habilidoso para realizar o projeto. \\
\hline 5 & I & Achei o projeto muito interessante. \\
\hline 6 & $\mathbf{P}$ & Fiquei tenso enquanto fazia o projeto. \\
\hline 7 & $\mathbf{C}$ & Acho que me saí muito bem nessa atividade, em comparação com outros estudantes. \\
\hline 8 & $\mathbf{I}$ & Realizar o projeto foi divertido. \\
\hline 9 & $\mathbf{P}$ & Fiquei relaxado enquanto fazia o projeto. (R) \\
\hline 10 & I & Gostei muito de fazer o projeto. \\
\hline 11 & $\mathbf{E}$ & Eu realmente não tive escolha sobre fazer o projeto. (R) \\
\hline 12 & $\mathbf{C}$ & Estou satisfeito com o meu desempenho nesta tarefa. \\
\hline 13 & $\mathbf{P}$ & Eu estava ansioso enquanto fazia o projeto. \\
\hline 14 & I & Eu pensei que o projeto era muito chato. (R) \\
\hline 15 & $\mathbf{E}$ & Senti que estava fazendo o que queria enquanto estava trabalhando no projeto. \\
\hline 16 & $\mathbf{C}$ & Eu me senti bastante hábil nesse projeto. \\
\hline 17 & $\mathbf{P}$ & Senti-me pressionado ao fazer o projeto. \\
\hline 18 & $\mathbf{E}$ & Eu senti que tinha que fazer o projeto. (R) \\
\hline 19 & $\mathbf{E}$ & Eu fiz a tarefa porque não tinha escolha. (R) \\
\hline 20 & $\mathbf{C}$ & Depois de trabalhar nessa tarefa por um tempo, me senti bastante competente. \\
\hline 21 & $\mathbf{C}$ & Essa foi uma atividade que não pude realizar muito bem. (R) \\
\hline
\end{tabular}

Tabela 1. Perguntas respondidas pelos estudantes ao final do curso.

A Figura 1 sumariza a média e o erro padrão das respostas (agregadas e separadas por sexo). Como pode-se observar na Fig. 1, nenhuma das dimensões analisadas apresentou diferença estatística entre os gêneros. No entanto, nota-se um alto IMI para o interesse na atividade enquanto pressão apresentou os menores índices. A confiança dos participantes em suas competências e que estavam fazendo uma tarefa que escolheram obteve resultados similares, com média 4,81 e 4,75, respectivamente. $\mathrm{O}$ alto índice em pressão pode ter sido motivado pelos curtos prazos para a execução dos projetos. 


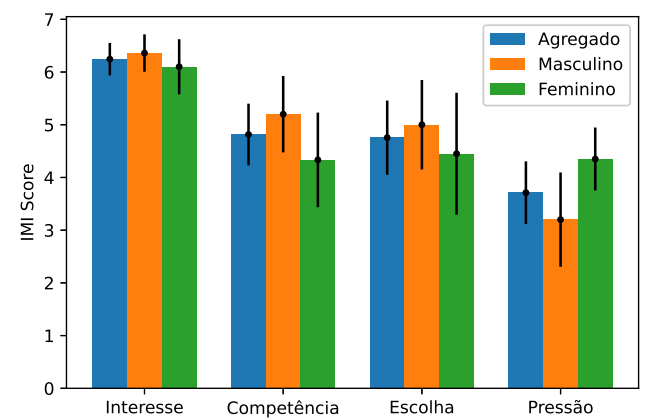

Figura 1. Índice de Motivação dos estudantes de PIC I.

\section{Conclusões}

Este trabalho apresentou um relato de experiência do desenvolvimento de um projeto de ensino voltado à integração hardware/software. Os artefatos produzidos ao longo do projeto foram disponibilizados publicamente e usados na disciplina de Projeto Integrado em Computação (PIC) para o segundo período de Engenharia da Computação. Importante destacar que a maioria dos projetos elaborados na disciplina utilizaram conceitos e técnicas não apresentados no curso ainda, mas que puderam ser abstraídos com ajuda dos roteiros utilizados. Por meio de um teste de Instrisic Motivation Index (IMI), observou-se que os projetos foram de alto interesse dos alunos e que eles/elas sentiam confiança em suas competências e escolhas. Embora consideremos que os curtos prazos contribuíram para o alto índice da pressão, compreender os reais motivos do que levou os estudantes a sentirem essa pressão é um trabalho futuro. No longo prazo, pretende-se acompanhar e comparar os índices de evasão na turma que participou deste projeto piloto.

\section{Referências}

Davok, D. F. and Bernard, R. P. (2016). Avaliação dos índices de evasão nos cursos de graduação da universidade do estado de santa catarina-udesc. Avaliação: Revista da Avaliação da Educação Superior (Campinas), 21(2):503-522.

Duch, B. J., Groh, S. E., and Allen, D. E. (2001). The power of problem-based learning: a practical" how to"for teaching undergraduate courses in any discipline. LLC.

Felipe, D. A. and de Melo Pinheiro, T. S. (2018). Seleção de tecnologias digitais para a gerência de projetos em disciplinas de projeto integrado. Rev. de Educação, Ciência e Tecnologia, 7(1).

INEP (2019). Censo na educação superior 2018. Instituto Nacional de Estudos e Pesquisas Educacionais Anísio Teixeira (Inep). Notas Estatísticas, 1(1).

Minerva, R., Biru, A., and Rotondi, D. (2015). Towards a definition of the internet of things (iot). IEEE Internet Initiative, 1(1):1-86.

Miorandi, D., Sicari, S., De Pellegrini, F., and Chlamtac, I. (2012). Internet of things: Vision, applications and research challenges. Ad hoc networks, 10(7):1497-1516.

Monteiro, V., Mata, L., and Peixoto, F. (2015). Intrinsic motivation inventory: Psychometric properties in the context of first language and mathematics learning. Psicologia: Reflexão e Crítica, 28(3):434-443.

UFPE (2016). Causas da evasão de alunos nos cursos de graduação presencial da UFPE. PróReitoria de Planejamento - Universidade Federal de Pernambuco, 1ed edition. 